\title{
FERRAMENTA PARA DETECÇÃO DE INCONFORMIDADES EM LIGAÇÕES DE MEDIDORES DE ENERGIA ELÉTRICA. A RELAÇÃO COM O APRENDIZADO NO ENSINO DA ENGENHARIA E A SOCIEDADE
}

\author{
DOI: 10.37702/2175-957X.COBENGE.2021.3702
}

Lucas Amorim Salvador Astini - I136594@dac.unicamp.br UNICAMP

R. Antônio João Tonela 190

13082-756 - Campinas - SP

Sergio Olivo - sergio.olivo@gmail.com

UNICAMP Universidade Estadual de Campinas

RUA CARL WILLIAN COOPER 316

08610-070 - SUZANO - SP

Leandro Tiago Manera - manera@fee.unicamp.br

UNICAMP

Av. Albert Einstein 400

13083-852 - Campinas - SP

Resumo: $O$ objetivo deste artigo é demonstrar que através de um método não intrusivo baseado na comparação dos valores das correntes lidas nos fios provenientes dos postes e das saídas dos medidores, além da análise dos ângulos de cada fase nas entradas dos medidores, há a possibilidade da detecção de furtos da energia elétrica. Tal estudo tem grande relação com o aprendizado na Engenharia, já que, para a detecção de fraudes para a captação de energia, é necessário o prévio conhecimento de matérias desenvolvidas na graduação, como a programação de microcontroladores, utilização da linguagem de programação HTML, CSS e JavaScript, ou seja, é essencial que as matérias oferecidas na graduação tenham sido entendidas, principalmente, as relacionadas com o campo de eletrônica e de computação para que artigos como este possam ser elaborados e áreas afins possam ser pesquisadas em conformidade com as mudanças constantes requeridas pela sociedade.

Palavras-chave: Medidores de energia. Detecção de fraudes. Microcontroladores. 


\section{(C. COBENGE e IV Simpósio Internacional de Educação em Engenharia

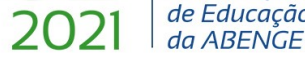 28 a 30 de SETEMBRO

Aprendizado da Engenharia. 


\section{FERRAMENTA PARA DETECÇÃO DE INCONFORMIDADES EM LIGAÇÕES DE MEDIDORES DE ENERGIA ELÉTRICA. A RELAÇÃO COM O APRENDIZADO NO ENSINO DA ENGENHARIA E A SOCIEDADE}

\section{INTRODUÇÃO}

O processo educacional, em geral, deve ser pauta de constantes discussões, pelo fato de ocorrerem intensas mudanças na sociedade, seja em âmbito político, tecnológico ou cultural. Frente a tais transformações há o surgimento da exigência de profissionais que conheçam as problemáticas de seu tempo e que sejam capazes de solucionar as demandas, desse modo, o ensino deve proporcionar aos alunos a capacidade de desenvolver soluções a partir dos conhecimentos a eles atribuídos, ou seja, tanto a educação quanto o futuro profissional devem estar atualizados quanto às soluções ansiadas pela sociedade. Diante deste prisma, Santos (2008) expõe que os processos de ensino estão progressivamente sendo discutidos em virtude das alterações que permeiam a atual sociedade brasileira.

Assim, diante das requisições atuais pela sociedade, um dentre tantos problemas a serem resolvidos e que esteja no âmbito no qual a Engenharia possa conceder sua cooperação, está a possibilidade do desenvolvimento de ferramentas capazes de detectar inconformidades nos medidores de energia elétrica. Tal procedimento é socialmente relevante pelo fato de que há com o furto grandes perdas de geração e distribuição de energia, o que é prejudicial tanto para os cidadãos, que muitas vezes pagam tarifas maiores para compensar os que não estão pagando corretamente (Smith, 2004), como também é danoso para a receita das companhias que a distribuem, além do fato de que os indivíduos que realizam o furto estão se submetendo a situações perigosas e estão incorrendo em crime almejando a redução do valor das contas de eletricidade, fato que ocorre desde domicílios a grandes empresas.

Sabe-se que diferentes procedimentos estão sendo utilizados para a obtenção da energia por meios ilícitos, desde contornar completamente os medidores, conectando a rede elétrica da infraestrutura diretamente à rede elétrica de baixa tensão, até a realização de alterações nos circuitos dos medidores a fim de alterar o seu funcionamento (Figura 1).

Figura 1 - Medidor eletrônico de energia adulterado.

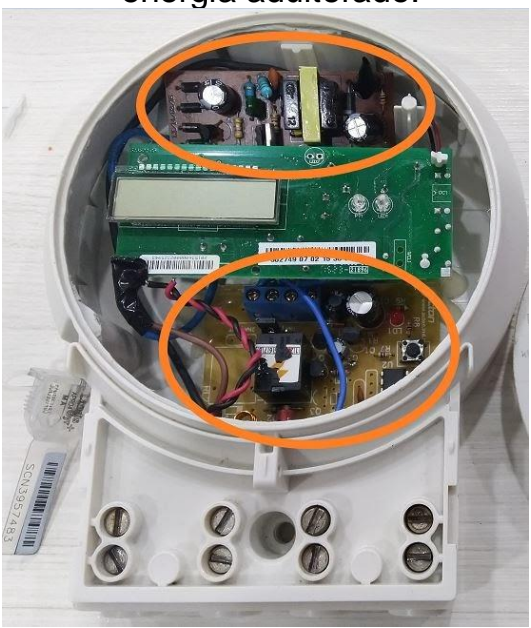

Fonte: Os autores. 
Assim, para tentar combater as citadas fraudes, entre outras, alguns métodos têm sido empregados, como o uso de medidores de energia inteligentes (Gao, 2019), ou até mesmo meios de detecção de elementos intrusivos por meio de radiofrequência (Astini, 2019). Analisa-se que diante das fraudes verificadas é necessário métodos de detecção que aliados com os estudos proporcionados pela graduação e a pesquisa amenizem o problema que há muito assola a sociedade com o furto da energia elétrica. Dessa maneira, neste artigo há a utilização de um procedimento não invasivo de inspeção que consiste na leitura e comparação das correntes das fases do poste e das saídas do medidor, além da leitura da tensão em cada fase do medidor (Figura 2). Tal método é capaz de identificar fraudes do tipo que contornam o medidor, popularmente conhecidas como "gatos" e também identificar alterações na ordem de conexão dos cabos de fase (sejam elas provenientes de uma alteração mal intencionada ou por erro de instalação).

Ressalta-se que a utilização do método mencionado pode ser uma importante ferramenta para o aprendizado em áreas como embarcado, eletrônica e microcontroladores, o que novamente evidencia que a educação deve ter seu plano de ensino formulado estrategicamente para que possa oferecer uma formação crítica e atual, para que os futuros profissionais se enquadrem na dinâmica social e cooperem, com sua área de formação, com as resoluções dos impasses contemporâneos.

Figura 2 - Setup de instalação.

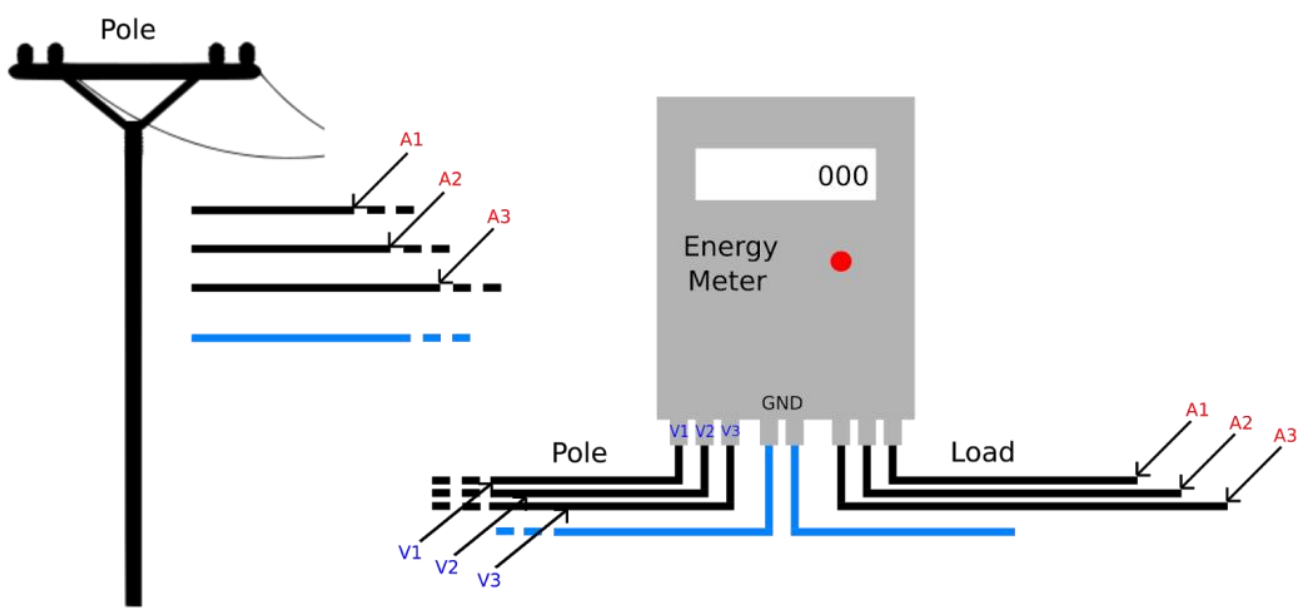

Fonte: Os autores.

Nesta imagem acima são indicados os pontos em que serão medidas as correntes, tanto nos fios do poste quanto nas saídas do medidor, além das tensões nas entradas do mesmo. Tais medições ocorrem conforme diagrama abaixo (Figura 3):

Figura 3 - Diagrama de instalação. 

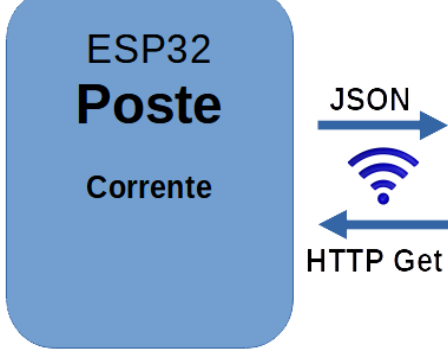

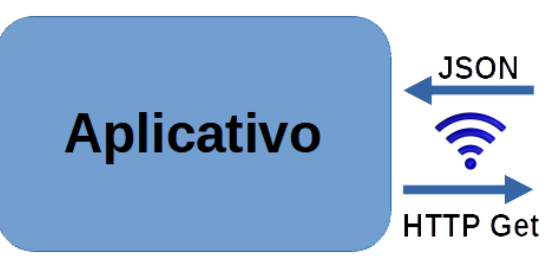

Fonte: Os autores.

\section{ESP32}

Medidor

Corrente

Tensão

Fator de Potência

Ângulo de Fase

Conforme o diagrama exibido, neste artigo haverá a produção de um sistema baseado no uso de microcontroladores (modelo ESP32) acoplados à rede elétrica de forma não invasiva, em que atuarão como multímetros medindo as correntes e tensões nas fases dos medidores de energia e as correntes nas fases dos fios no poste, além de calcularem a potência real, a potência aparente, o fator de potência e o ângulo de cada fase. Estes sistemas devem então enviar os dados das medidas através de uma rede Wi-Fi para um dispositivo operando um aplicativo HTML. Tal aplicativo é responsável por analisar estes dados e disponibilizá-los aos usuários. Nota-se aqui a oportunidade para o aprendizado do uso de sistemas embarcados em uma aplicação real.

Tendo sido estabelecido esse ecossistema é possível inferir conclusões sobre a existência de inconformidades com a instalação elétrica, além de ser possível monitorar a qualidade da energia sendo distribuída. Ademais, é imprescindível a atenção para o fato de que o aprendizado em programação e aplicativos é de suma importância, pois ele possibilitou o desenvolvimento e o progresso da pesquisa, portanto, tanto os professores, que são os mediadores do conhecimento, quanto o aluno desenvolvedor da pesquisa, merecem o reconhecimento, porém sabe-se que é o aluno que assume a posição de protagonista de seu próprio aprendizado (DE SOUZA, 2009; SANTOS et al., 2019).

\section{REVISÃO BIBLIOGRÁFICA}

Diferentes métodos estão sendo usados para furtar energia, que vão desde contornar completamente o medidor, conectando a rede elétrica da casa diretamente à rede elétrica de baixa tensão, até fazer alterações nos circuitos do medidor de energia.

Assim, o Quadro 1 apresenta alguns estudos sobre diferentes métodos que têm sido usados para detectar ou evitar o furto de energia.

Quadro 1: Métodos de detecção de fraudes.

\begin{tabular}{|c|c|}
\hline Autores & Método \\
\hline Y. Gao, B. Foggo, N. Yu & Medidor de energia inteligente (Gao, 2019) \\
\hline $\begin{array}{c}\text { Manera, M. Bressani, T. B. Siqueira, V. Gialluca, } \\
\text { J.M. Santos }\end{array}$ & $\begin{array}{c}\text { Detecção de fraude por meio de técnica não } \\
\text { intrusiva utilizando compatibilidade eletromagnética } \\
\text { (Astini, 2019) }\end{array}$ \\
\hline S. Sahoo, D. Nikovski, T. Muso, K. Tsuru & Medidor de energia inteligente (Sahoo, 2015) \\
\hline R.Jiang, R. Lu, Y. Wang, J. Luo, C. Shen & $\begin{array}{c}\text { Rede de distribuição de energia inteligente (Jiang, } \\
\text { 2014) }\end{array}$ \\
\hline S. S. Bhalshankar, C. S. Thora & $\begin{array}{c}\text { Rede de distribuição de energia inteligente e Direct } \\
\text { Hooking (Bhalshankar, 2017) }\end{array}$ \\
\hline
\end{tabular}

Fonte: Os autores. 


\section{METODOLOGIA}

Além da etapa técnica para a construção da pesquisa voltada para detecção de inconformidades em ligações de medidores de energia elétrica foi realizada uma pesquisa com intuito de relacionar o ensino de Engenharia com este artigo, o que provou-se muito importante, pois há o aguçamento quanto ao aprendizado e a resolução das questões sociais presentes, já que, busca-se através das matérias aprendidas na Engenharia a colaboração com as requisições dos cidadãos e até mesmo do ambiente em que se vive. Diante disso, Hoehnke, Koch e Lutz (2005) afirmam que os métodos tradicionais de ensino orientam os alunos no sentido da negligência da criatividade com menos capacidade para a nova realidade, portanto, depreende-se que a sociedade necessita de indivíduos que sejam adaptáveis e que assim, os conteúdos a eles passados também.

Seguindo-se com a metodologia da pesquisa, neste artigo estão sendo utilizados dois microcontroladores modelo ESP32. Um para coletar dados das correntes do poste e outro para as correntes e tensões no medidor. Em ambos estão implementados WebServers que respondem a chamadas HTTP GET realizadas através de métodos assíncronos (AJAX - Asynchronous Javascript XML) e formatadas em JSON (JavaScript Object Notation), como esquematizado na Figura 4. A comunicação é realizada por meio de uma conexão Wi-Fi utilizando o recurso de Hotspot presente nos Smartphones.

Figura 4: Método de funcionamento.
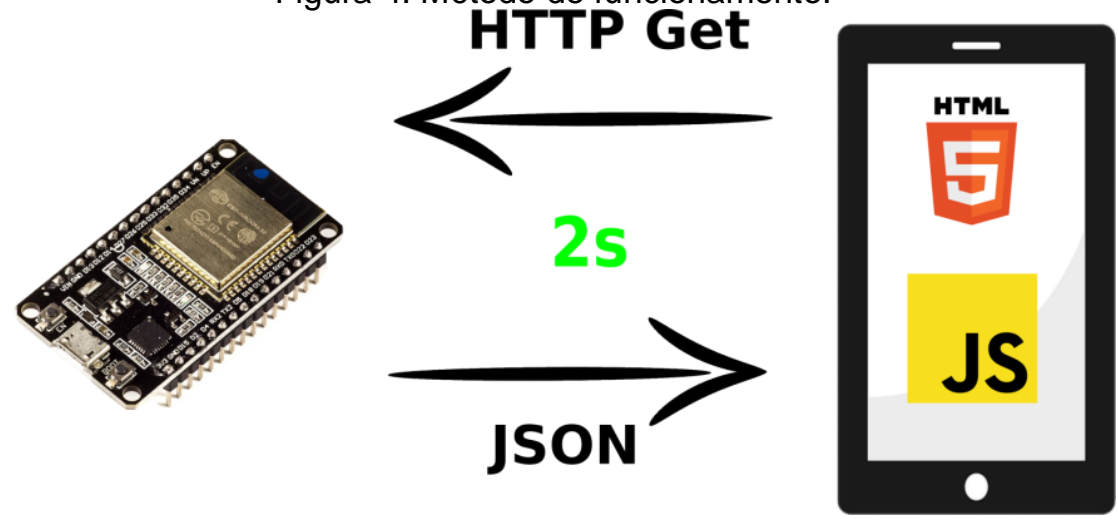

Fonte: Os autores.

As requisições são feitas por uma aplicação Cliente desenvolvida em HTML5 e Javascript rodando em um Smartphone. Esse programa faz o requerimento dos arquivos JSON a cada 2 segundos e os analisa por meio do AJAX. As informações são então disponibilizadas tanto numericamente como de forma gráfica, facilitando assim a visualização (Figura 5). Além disso, o programa conta com a opção de download dos dados coletados, gerando assim, um arquivo no formato CSV.

Por isso, até esse ponto, podemos notar que tal aplicação percorre tanto as experiências (e aprendizado) em embarcado e programação.

Figura 5: Aplicativo (HTML5). 


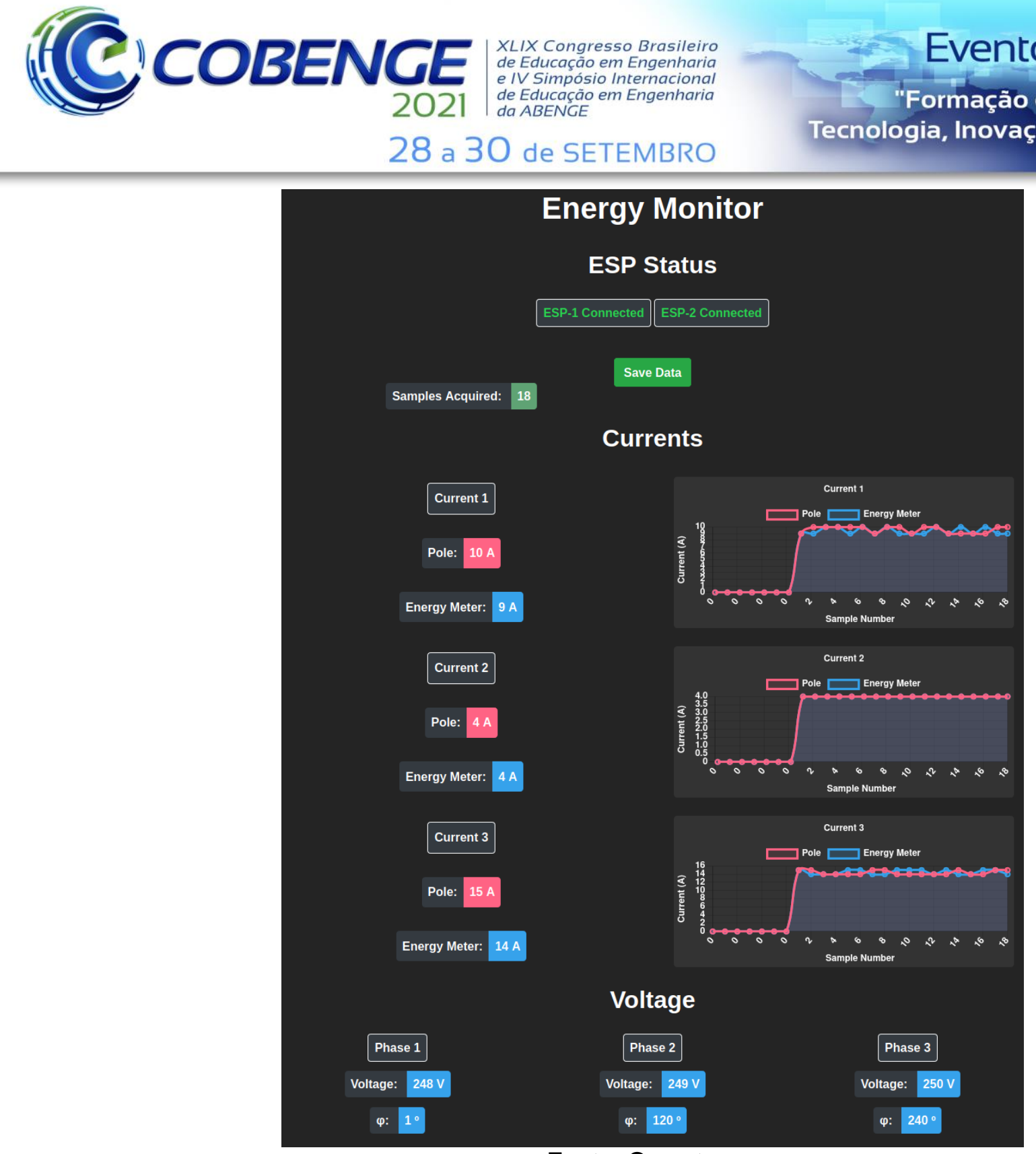

Fonte: Os autores.

A imagem acima apresenta a interface do aplicativo HTML. Nela são apresentados os valores instantâneos das correntes, tensões, ângulos das fases e gráficos contendo as variações das correntes ao longo das medições. Na parte superior há a indicação de quantas medições foram realizadas (Samples Acquired) e um botão (Save Data) que reúne essas medições e as disponibiliza para download, gerando um arquivo CSV.

\section{HARDWARE}

Como já referido, neste artigo estão sendo utilizados 2 microcontroladores modelo ESP32. Estes possuem como diferencial a presença de antenas de $2,4 \mathrm{GHz}$ permitindo que se conectem às redes Wi-Fi. Outro aspecto importante é o limite de tensão suportado pelas portas do microcontrolador. Nestes modelos, as entradas suportam tensões entre $0 \mathrm{~V}$ e 3,3 V. Dessa forma, é preciso não apenas reduzir a amplitude das ondas de tensões que serão lidas, mas também gerar um offset para que as mesmas não apresentem valores negativos.

\subsection{Sensor de corrente AC}

Para medir as correntes dos fios do poste e da saída do medidor de energia, de forma não invasiva, é indispensável o uso de um sensor de corrente que funcione como um transformador (Figura 6). 
Figura 6: Transformador de corrente com resistor interno.

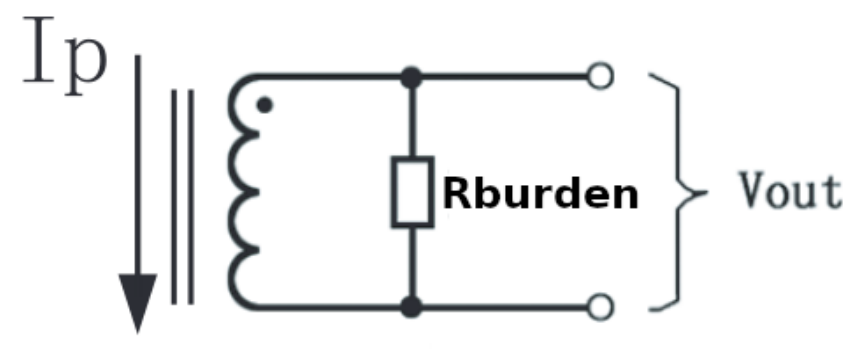

\section{Voltage output type}

Fonte: Modificado de YHDC, http://en.yhdc.com.

Neste método, o campo magnético proveniente da corrente que passa pelo fio induz corrente no enrolamento do sensor. Como as portas do microcontrolador apenas medem tensões, utiliza-se então um resistor (Rburden) para que possa ser medida a tensão sobre o mesmo, permitindo assim, -pela relação de espiras deste transformador- calcular a corrente que atravessa o fio. Neste projeto estão sendo utilizados sensores modelos SCT013-000, como mostra a Figura 7.

Figura 7: Sensor de corrente não invasivo.

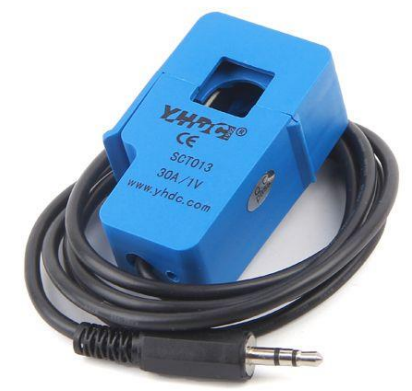

Fonte: YHDC, http://en.yhdc.com.

Este modelo possui um resistor interno (Rburden) de 60 Ohms e uma relação de espiras de 1800:1. Devido às limitações das entradas da ESP32 (não suportando tensões negativas e nem acima de 3,3V) foi utilizado um divisor de tensão com resistores de valores altos, no caso $470 \mathrm{kOhm}$, visando não produzir uma grande corrente, para gerar um offset DC na onda da tensão medida sobre o Rburden. Esse divisor foi alimentado pelas saídas 3,3V e GND do microcontrolador. Dada as características do sensor de corrente utilizado, a maior corrente que esse sistema consegue medir é de $30 \mathrm{~A}$ (RMS), o que resulta em 23,5 mA (de pico) no secundário do sensor. Assim, ao medir essa corrente máxima, temos a seguinte onda de tensão sendo lida por uma das entradas do microcontrolador (Figura 8):

Figura 8: Esquemático do sensor de corrente. 

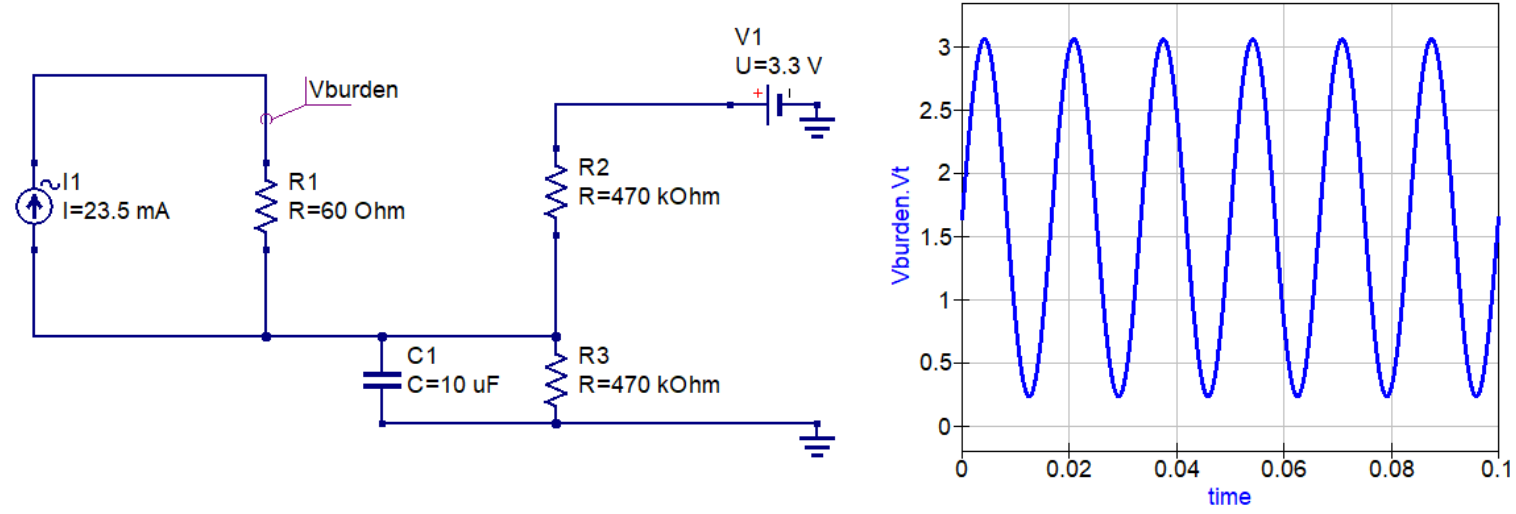

Fonte: Os autores.

Esta imagem mostra a tensão (em volts) lida pela entrada (input) do microcontrolador e como pode ser observado, as tensões de pico encontram-se dentro dos limites de $0 \mathrm{~V}$ a 3,3 $\mathrm{V}$ do dispositivo. Tal onda possui frequência de $60 \mathrm{~Hz}$ (mesma frequência da onda sendo lida pelo sensor de corrente).

\subsection{Sensor de tensão AC}

Para as medições das tensões das fases, um princípio bastante semelhante ao da medição de corrente é utilizado. Como visto anteriormente, é necessário reduzir a amplitude da onda de tensão além de introduzir um offset a fim de que não possua valores negativos, satisfazendo assim, os requisitos do microcontrolador. Para isso, novamente é utilizado um transformador para reduzir a amplitude da onda (juntamente com o auxílio de um divisor de tensão). Já para o offset, um circuito de divisão de tensão idêntico ao utilizado no sensor de corrente é empregado. O esquemático deste sensor de tensão, com um transformador de $6 \mathrm{~V}$ no secundário como exemplo, pode ser observado abaixo (Figura 9):

Figura 9: Esquemático do sensor de tensão.

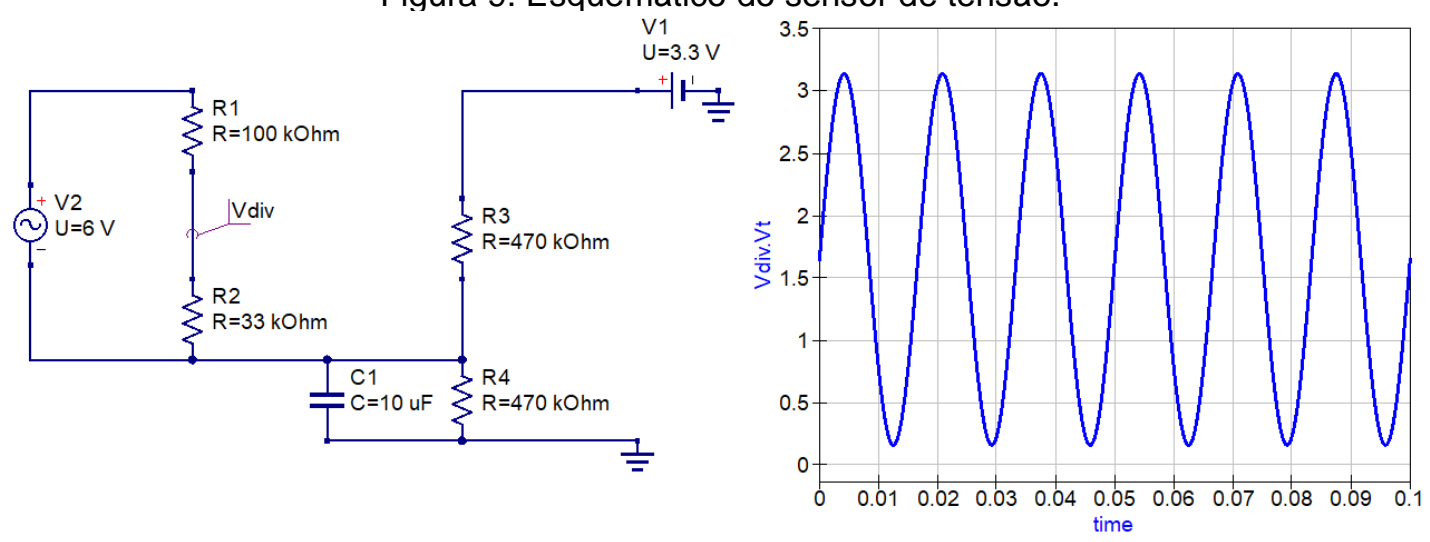

Fonte: Os autores.

Assim como no caso do sensor de corrente, esta imagem mostra a onda de tensão (em volts) lida pela entrada (input) do microcontrolador e a mesma encontra-se dentro dos limites de $0 \mathrm{~V}$ a 3,3 $\mathrm{V}$ do dispositivo. Tal onda também possui frequência de $60 \mathrm{~Hz}$ (mesma frequência da tensão que passa pelo enrolamento primário do transformador).

\section{SOFTWARE}

Fica claro então que hoje o aprendizado em engenharia deve alinhar os conhecimentos em embarcados, programação e eletrônica. Este projeto, portanto, pode ser 
um ótimo exemplo para uma aplicação prática de atividades de ensino para desenvolver os conhecimentos nessas diversas áreas do ensino.

\subsection{Microcontroladores}

Para conectar a ESP32 a uma rede Wi-Fi, está sendo utilizada a biblioteca "WiFi.h". Optou-se por utilizar IPs fixos, com isso o aplicativo mais facilmente consegue identificar os microcontroladores.

Referente às medições das correntes e das tensões, este projeto utiliza a biblioteca "EmonLib.h". A partir desta, é possível medir os valores de corrente e tensão, bem como calcular a potência aparente, potência real e o fator de potência.

Todas as medições e cálculos são realizados quando o microcontrolador recebe uma requisição do tipo HTTP GET. Para isso, está configurado um WebServer que atende essas requisições fornecendo os dados no formato JSON (Figura 10), utilizando a biblioteca "ArduinoJson.h".

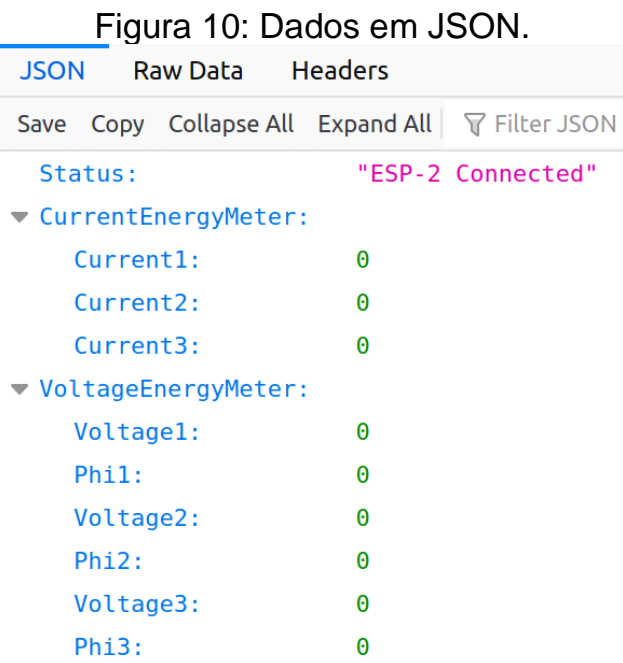

Fonte: Os autores.

\subsection{Aplicativo HTML}

Pretendendo abranger maior variedade de dispositivos compatíveis, este aplicativo foi escrito utilizando as linguagens HTML, CSS e JavaScript. Dessa forma, qualquer dispositivo contendo um browser é capaz de executar esse programa.

Sua estrutura gráfica foi feita a partir do framework Bootstrap, o que permite a divisão da tela em até 12 segmentos horizontais (a cada linha), além de conter formulários, botões, tabelas, carrosséis de imagens e outros. Essa ferramenta é importante para a adequação do conteúdo ao display utilizado, de forma que ele automaticamente organiza a disposição do conteúdo na tela conforme variação horizontal (Figura 11).

Figura 11: Diferentes organizações dos conteúdos da página utilizando Bootstrap. 


\section{COBENCE de Educação em Engenharia da $A B E N G E$ \\ 28 a 30 de SETEMBRO}
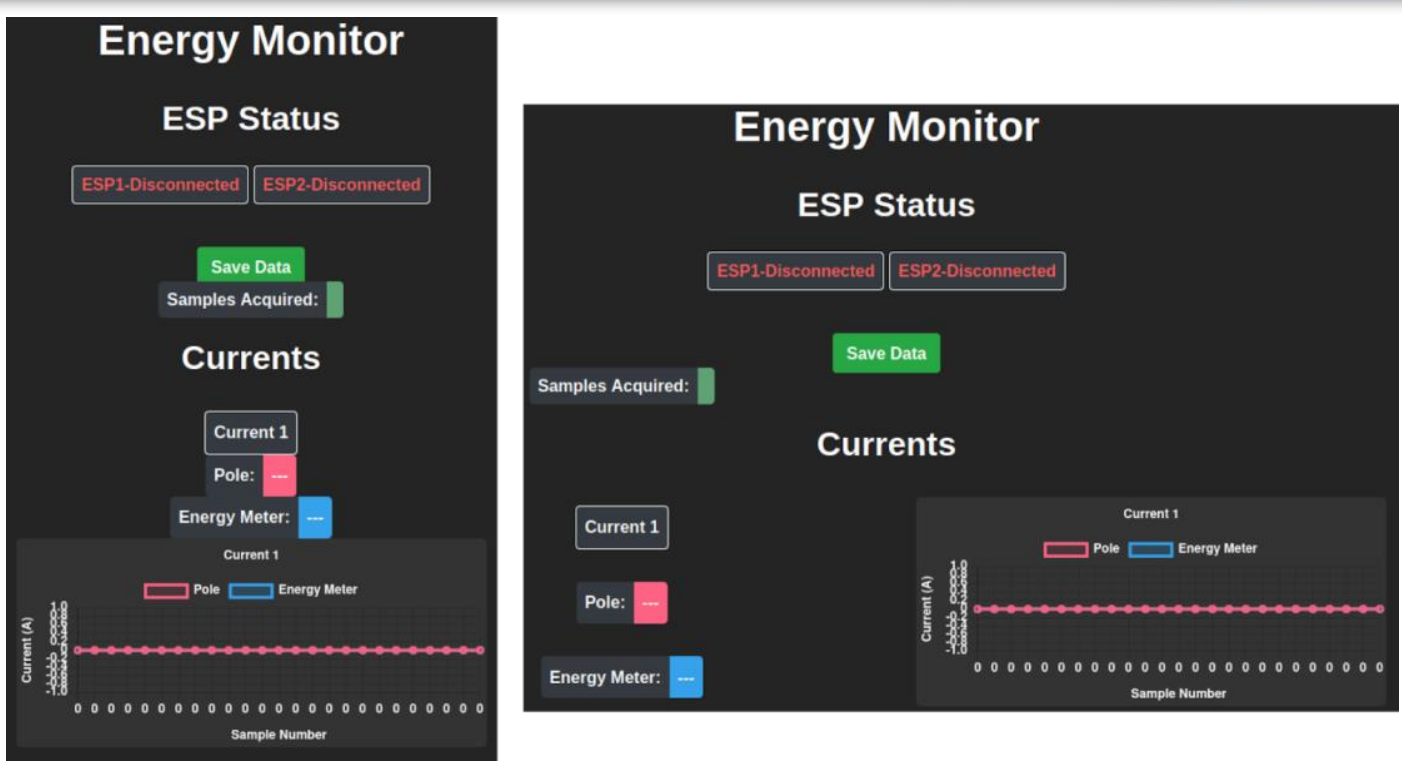

Fonte: Os autores.

As informações exibidas são obtidas por meio de requisições do tipo HTTP GET enviadas para os 2 microcontroladores, recebendo como resposta arquivos JSON. Essas requisições e suas interpretações são realizadas por meio do AJAX. Os dados são então apresentados no HTML, contando com gráficos para facilitar a visualização.

\section{RESULTADOS}

Para realizar os testes deste projeto foi criado um circuito alimentado por uma fase de $127 \mathrm{~V}$ e um neutro, contendo 4 cargas resistivas idênticas, no caso 4 lâmpadas halógenas. Para medir a diferença de corrente constatada pelo medidor em relação ao poste no caso da existência de um bypass, foi introduzida uma ligação do fio de fase antes do medidor até um interruptor paralelo. Este interruptor tem a função de chavear entre o bypass e a saída do medidor, alimentando assim, as 2 lâmpadas superiores. Dessa forma, quando chaveado na posição "medidor", as 4 lâmpadas são alimentadas pela fase proveniente do medidor, mas ao chavear o interruptor na posição "bypass", 2 lâmpadas continuam alimentadas pela fase vinda do medidor (2 lâmpadas da parte de baixo) enquanto as 2 superiores são alimentadas pelo bypass, contornando o medidor. O setup foi montado conforme mostrado abaixo (Figura 12):

Figura 12: Setup de testes.

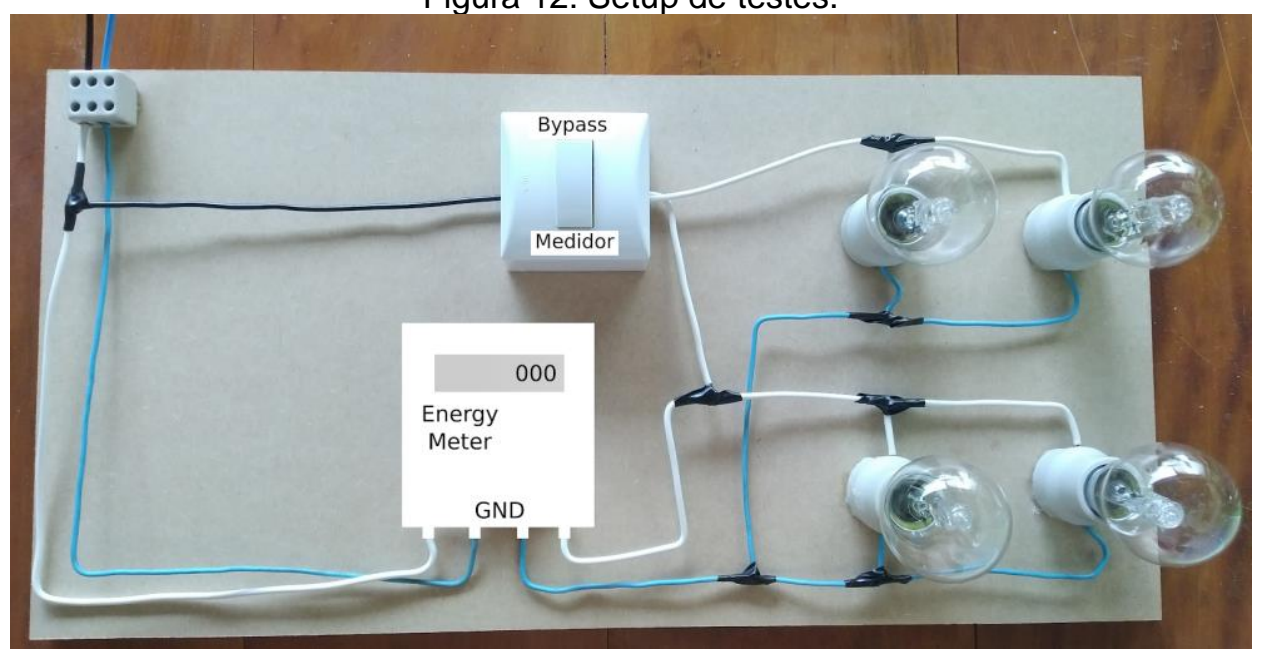


Fonte: Os autores.

Testes com este setup resultaram nas seguintes medições (Figura 13):

Figura 13: Funcionamento do sistema.

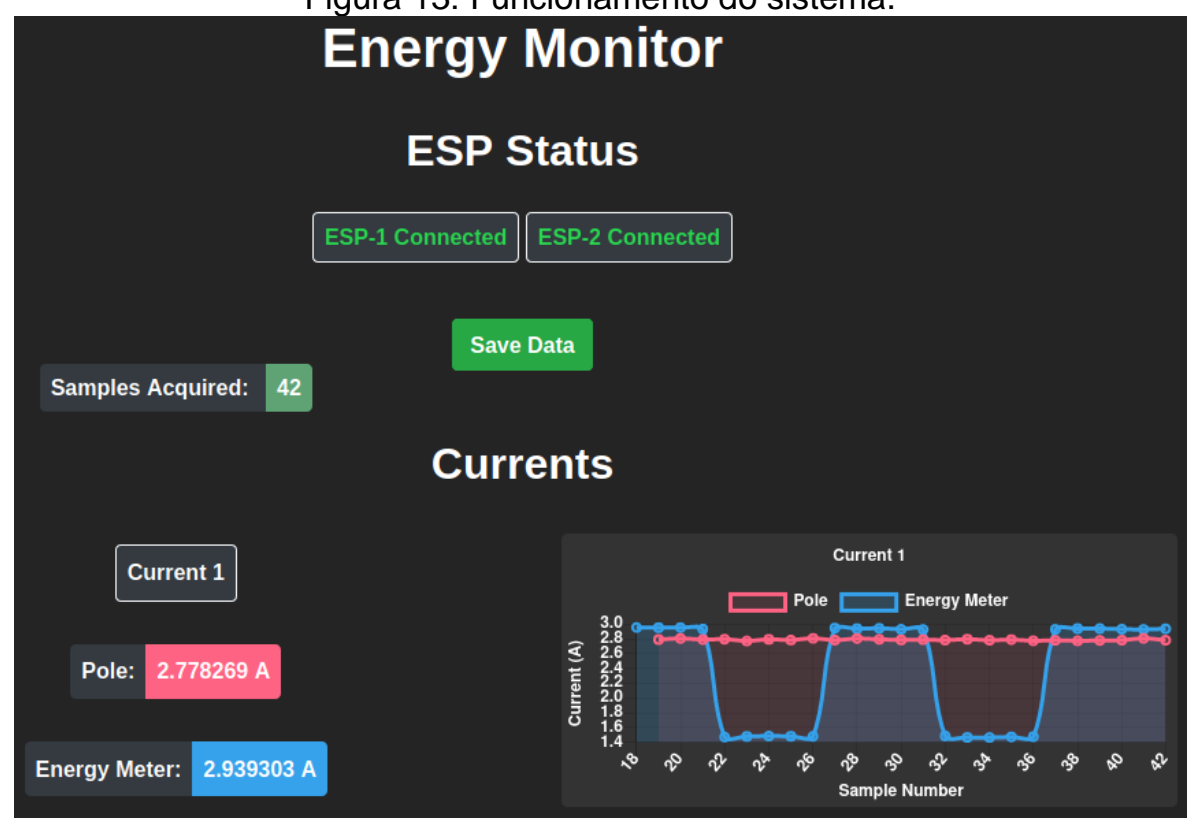

Fonte: Os autores.

Como já supracitado, esse sistema é capaz de identificar pequenas variações de corrente (no caso a diferença de corrente proveniente de 2 lâmpadas). Sendo assim, é uma forma bastante eficaz de fiscalizar a existência de inconformidades nas instalações elétricas. Além disso, apesar deste exemplo conter informações apenas de uma fase, esse sistema é escalonável para instalações trifásicas.

Por fim, o artigo tem o intuito também de estimular a educação da Engenharia voltada para a resolução dos mais diversos problemas sociais atuais, incentivando estratégias ativas de aprendizagem que promovam também as competências transversais dos indivíduos juntamente com a aplicação de seu conhecimento teórico na prática. Verifica-se que a educação se encontra vinculada ao projeto de cidadania (DIAS, 2019), assim fica claro que os conhecimentos passados devem ser desenvolvidos de acordo com as exigências do sistema, ou seja, deve haver uma constante atualização no conteúdo passado para os alunos para o progresso social.

\section{Agradecimentos}

$\mathrm{O}$ presente trabalho foi realizado com apoio do CNPq, Conselho Nacional de Desenvolvimento Científico e Tecnológico - Brasil.

\section{REFERÊNCIAS}

Astini, Lucas Amorim Salvador et al. Use of Electromagnetic Compatibility Techniques for Analyses of Intrusive Elements in Energy Meters. IEEE International Autumn Meeting on Power, Electronics and

Computing

(ROPEC), 2019.

Bhalshankar, Surekha S.; Thorat, C. S. MAHADISCOM electrical theft controlling mechanism: (Smart grid advanced metering infrastructure and drone operated technology for controlling 
theft by direct hooking). International Conference on Wireless Communications, Signal Processing and Networking (WiSPNET), 2017.

DIAS, E, \& PINTO, F.C.F. 2019. Ensaio: Avaliação e Políticas Públicas em Educação. SCIELO, pág 2-3.

DOS SANTOS, C. A. M., PEREIRA, M. A. C. BARRETO, M. A. M., DE SOUZA, M. A., \& CICARELLI, P. O. 2019. CEMTRAL: uma nova metodologia híbrida de ensino e aprendizagem. Revista Brasileira de Aprendizagem Aberta e a Distância, 18(1), 18-18.

Gao, Yuanqi; Foggo, Brandon; Yu, Nanpeng. A Physically Inspired Data-driven Model for Electricity Theft Detection with Smart Meter Data. IEEE Transactions on Industrial Informatics, 2019.

Jiang, Rong et al. Energy-Theft Detection Issues for Advanced Metering Infrastructure in Smart $\begin{array}{lllll}\text { Grid. Tsinghua } & \text { Science }\end{array}$

Sahoo, Sanujit et al. Electricity theft detection using smart meter data. IEEE Power \& Energy Society Innovative Smart Grid Technologies Conference (ISGT), 2015.

Smith, Thomas B. Electricity theft: a comparative analysis. Elsevier Journal Energy Policy, Vol. $32, \quad$ no. $18, \quad$ pp. 2004.

\title{
TOOL FOR DETECTION OF INCONFORMITIES IN CONNECTIONS OF ELECTRIC ENERGY METERS. THE RELATIONSHIP WITH LEARNING IN TEACHING IN ENGINEERING AND SOCIETY
}

\begin{abstract}
The objective of this article is to demonstrate that through a non-intrusive method based on the comparison of the values of the currents read in the wires coming from the posts and the meter exits, in addition to the analysis of the angles of each phase at the meter inputs, there is the possibility of detecting theft of electricity. Such study has a great relationship with the learning in Engineering, since, for the detection of frauds it is necessary the previous knowledge of subjects developed in the graduation, as the programming of microcontrollers, use of the programming language HTML, CSS and JavaScript, therefore, it is essential that the subjects offered in the graduation course have been understood so that articles like this can be elaborated and related areas can be researched in accordance with the constant changes required by society.
\end{abstract}

Keywords: Energy meters. Fraud detection. Microcontrollers. Engineering Learning. 